University of Nebraska - Lincoln

DigitalCommons@University of Nebraska - Lincoln

$12-2-2018$

\title{
The transcriptomes of the cattle parasitic nematode Ostertagia ostartagi
}

Sahar Abubucker

Dante S. Zarlenga

John Martin

Yong Yin

Zhengyuan Wang

See next page for additional authors

Follow this and additional works at: https://digitalcommons.unl.edu/usdaarsfacpub

This Article is brought to you for free and open access by the U.S. Department of Agriculture: Agricultural Research Service, Lincoln, Nebraska at DigitalCommons@University of Nebraska - Lincoln. It has been accepted for inclusion in Publications from USDA-ARS / UNL Faculty by an authorized administrator of DigitalCommons@University of Nebraska - Lincoln. 


\section{Authors}

Sahar Abubucker, Dante S. Zarlenga, John Martin, Yong Yin, Zhengyuan Wang, James P. McCarter, Louis Gasbarree, Richard K. Wilson, and Makedonka Mitreva 


\title{
The transcriptomes of the cattle parasitic nematode Ostertagia ostartagi
}

\author{
Sahar Abubucker ${ }^{\mathrm{a}}$, Dante S. Zarlenga ${ }^{\mathrm{b}}$, John Martin ${ }^{\mathrm{a}}$, Yong Yin ${ }^{\mathrm{a}}$, Zhengyuan Wang ${ }^{\mathrm{a}}$, \\ James P. McCarter ${ }^{\mathrm{a}, \mathrm{c}}$, Louis Gasbarree ${ }^{\mathrm{b}}$, Richard K. Wilson ${ }^{\mathrm{a}}$, Makedonka Mitreva ${ }^{\mathrm{a}, *}$ \\ ${ }^{a}$ The Genome Center, Department of Genetics, Washington University School of Medicine, St. Louis, MO 63108, United States \\ ${ }^{\mathrm{b}}$ Bovine Functional Genomics Laboratory and Animal Parasitic Diseases Laboratory and U.S. National Parasite Collection, U.S. Department of Agriculture, \\ Beltsville, MD 20705, United States \\ ${ }^{\mathrm{c}}$ Divergence Inc., 893 North Warson Road, St. Louis, MO 63141, United States
}

\section{A R T I C L E I N F O}

\section{Article history:}

Received 2 December 2008

Received in revised form 13 February 2009

Accepted 13 February 2009

\section{Keywords:}

Cattle

Parasite

Nematode

Transcripts

ESTs

Ostertagia ostertagi

Comparative genomics

\begin{abstract}
A B S T R A C T
Ostertagia ostertagi is a gastrointestinal parasitic nematode that affects cattle and leads to a loss of production. In this study, we present the first large-scale genomic survey of $O$. ostertagi by the analysis of expressed transcripts from three stages of the parasite: thirdstage larvae, fourth-stage larvae and adult worms. Using an in silico approach, 2284 genes were identified from over 7000 expressed sequence tags and abundant transcripts were analyzed and characterized by their functional profile. Of the 2284 genes, 66\% had similarity to other known or predicted genes while the rest were novel and potentially represent genes specific to the species and/or stages. Furthermore, a subset of the novel proteins were structurally annotated and assigned putative function based on orthologs in Caenorhabditis elegans and corresponding RNA interference phenotypes. Hence, over $70 \%$ of the genes were annotated using protein sequences, domains and pathway databases. Differentially expressed transcripts from the two larval stages and their functional profiles were also studied leading to a more detailed understanding of the parasite's life-cycle. The identified transcripts are a valuable resource for genomic studies of 0 . ostertagi and can facilitate the design of control strategies and vaccine programs.
\end{abstract}

(c) 2009 Elsevier B.V. All rights reserved.

\section{Introduction}

Ostertagiosis is caused by the nematode Ostertagia ostertagi, a stomach parasite of cattle found predominantly in temperate regions of the world. Ostertagia belongs to the order Strongylida, which includes numerous livestock parasites and hookworms (Ancylostoma and Necator species), and is part of clade $\mathrm{V}$ along with the extensively

\footnotetext{
Nucleotide sequence data reported in this paper and sequenced at the Washington University's Genome Center are available in the GenBank, EMBL and DDBJ databases. The accession numbers for the Ostertagia ostertagi ESTs are 19379209-19381350, 12652777-21653605, 21265177-21265790, 20130441-20133438.

* Corresponding author at: 4444 Forest Park Boulevard, St. Louis, MO 63108, United States. Tel.: +1 314286 2005; fax: +1 3142861810.

E-mail address: mmitreva@watson.wustl.edu (M. Mitreva).
}

characterized free-living nematode Caenorhabditis elegans (Blaxter et al., 1998). O. ostertagi eggs are passed within the host from the female worm, finally reaching the dung where they hatch to the first larval stage (L1), and then develop to the second larval stage (L2). The infective third larval stage (L3) has a protective layer that enables it to survive long periods in the environment and also enables mobility for migration and dissemination on pasture. After host ingestion, the L3 lose their protective sheath and burrow into the gastric glands of the abomasum where they develop to fourth-stage larvae (L4). The L4 can proceed directly to adult worms at 3-4 weeks post infection or enter seasonal arrested development for up to 6 months (diapause). The mechanism of this arrest is unknown. In C. elegans, arrest as a non-feeding dauer larvae (L3d) is triggered in the L1/early L2 by adverse conditions for survival such as lack of food, overcrowding or environmental extremes (Riddle and 
Albert, 1997). It is not yet known whether L4 arrest in a trichostrongyle-like 0 . ostertagi has any ancestral connection or shared mechanisms with the C. elegans L3d.

Type I ostertagiosis occurs when the adult worm proceeds directly through the larval stages without any inhibition. Type II ostertagiosis occurs when the L4 larvae resume development after a period of seasonal arrest. Surprisingly, seasonal arrest is regional where some L4 become developmentally inhibited in the spring and summer, and in less temperate regions of the world they become inhibited in the fall and winter. Both types of ostertagiosis cause damage to the host abomasum and are accompanied by weight loss and severe diarrhea that can lead to a loss of productivity and increased morbidity and mortality. Infections with the entire class of gastrointestinal (GI) nematodes cost the cattle producers hundreds of millions of dollars annually.

Anthelmintic drugs are typically used to treat ostertagiosis. Although evidence for resistance to these drugs has yet to surface in 0 . ostertagi, it is nonetheless on the increase among this broad group of GI nematodes including Haemonchus, Cooperia, and Trichostrongylus (Wolstenholme et al., 2004). O. ostertagi infection does not rapidly confer natural immunity to re-infection. Other modes of intervention such as vaccination with Ostertagia polyprotein products (Vercauteren et al., 2004) and cysteine proteinase (Geldhof et al., 2002) have been investigated for preventing infections though results have been equivocal. This study has generated the first extensive genomics resource for 0 . ostertagi. The information offers insights into mechanisms associated with the host-parasite interface and also the parasite's life-cycle which may accelerate progress towards identification of effective targets and strategies for parasite control.

\section{Material and methods}

\subsection{O. ostertagi material, RNA extraction, and library construction}

Third-stage exsheathed larvae were collected from 14day fecal-sphagnum moss cultures of 0 . ostertagi eggs. The larvae were recovered by overnight passage on a Baermann apparatus, and then cleaned by passage through a $20-\mu \mathrm{m}$ nylon mesh. The larvae were then subjected to a treatment with $1.25 \%$ chlorox to induce excystation. The larvae were washed with 5 changes of PBS and then pelleted and snap frozen in liquid nitrogen.

Late $\mathrm{L} 4$ were recovered 10 days after infection of young calves with 0 . ostertagi $\mathrm{L} 3$. The calves were killed and the abomasa (gastric glands) were recovered and washed extensively and then placed in a Baermann apparatus containing warm PBS for approximately $4 \mathrm{~h}$. The L4 that migrated from the tissues were washed extensively, pelleted and snap frozen in liquid nitrogen.

Pulverization was performed using an Alloy Tool Steel Set (Fisher Scientific International). Total RNA of L3 and L4 was prepared using TRIzol reagent (GibcoBRL, Life Technologies). Two types of cDNA libraries were constructed from each stage: SMART based (Mitreva et al., 2005) and SL1-1 based (Mitreva et al., 2004a).

\subsection{EST sequencing and clustering}

EST processing and clustering were performed as described (McCarter et al., 2003; Mitreva et al., 2004c). Submissions have been deposited to GenBank, and information for clone requests is available at www.nematode.net (Martin et al., 2009). One hundred and five ESTs were defined as chimeras and excluded from the analysis. Later examination of the 0 . ostertagi culture used as a source for the L3 sample identified a minority population of Cooperia oncophora worms, a very closely related trichostrongylid intestinal nematode that frequently co-infects hosts along with 0 . ostertagi. The $O$. ostertagi culture used for the L4 samples was found not to have any $C$. oncophora worms. To determine the extent of $C$. oncophora ESTs in the O. ostertagi sample in silico, all L3and L4-biased and -specific clusters from the sample were screened against $857 \mathrm{C}$. oncophora and 186 O. ostertagi sequences retrieved from NCBI (June 2008) using WUBLAST. As orthologs might not possibly exist in the NCBI database for both species, we analyzed the subset of the L3-enriched genes that had homology to both species (with $E$-value better than $1 \mathrm{e}^{-05}$ ). We identified two genes (with 99 and 2 ESTs respectively) out of nine that were more homologous to $C$. oncophora than $O$. ostertagi and the remaining genes showed stronger identity with $O$. ostertagi. This analysis was repeated with L4-enriched contigs to evaluate the incidence of $C$. oncophora. From the L4 set, five genes showed sequence identity with $C$. oncophora and $O$. ostertagi and only one of them had stronger matches to the $C$. onchophora homologs. In both analyses (L3 and L4), whenever the hits were better to $C$. oncophora the subject was different units of the cytochrome oxidase, and the second best hit was to $O$. ostertagi. The 0 . ostertagi hit was with higher percentage identity but shorter alignment which resulted in a better $e$-value for the $C$. onchophora hit. This analysis indicates that we cannot confirm that $C$. oncophora transcripts are present in the 0 . ostertagi L3 sample and if they are, their presence is not substantial, and furthermore, given preliminary evidence of genetic and biological similarities among these trichostrongyles, it is unlikely that small amounts of contamination would skew the overall results. In addition, in cases where clusters comprised of ESTs from multiple stages, this concern is eliminated as contamination was not an issue with the L4 and the adult sample (Fig. 1).

Expressed Sequence Tags (ESTs) (6557) from L3, L4, and the adult stages were submitted to GenBank. Additional ESTs (485) were downloaded from GenBank on 01/03/ 2006. NemaGene clustering was performed on ESTs from the L3, L4 and adult worms. This clustering groups all ESTs into contigs representing transcripts with potential isoforms. Contigs that are mostly identical are then grouped into unique clusters (McCarter et al., 2003). Rarely, clusters may also include highly related gene family members. After quality control and screening, 7042 ESTs were organized into contigs which were translated using prot4EST (Wasmuth and Blaxter, 2004). There were 4605 contigs with valid translations that clustered into 2284 putative genes. 


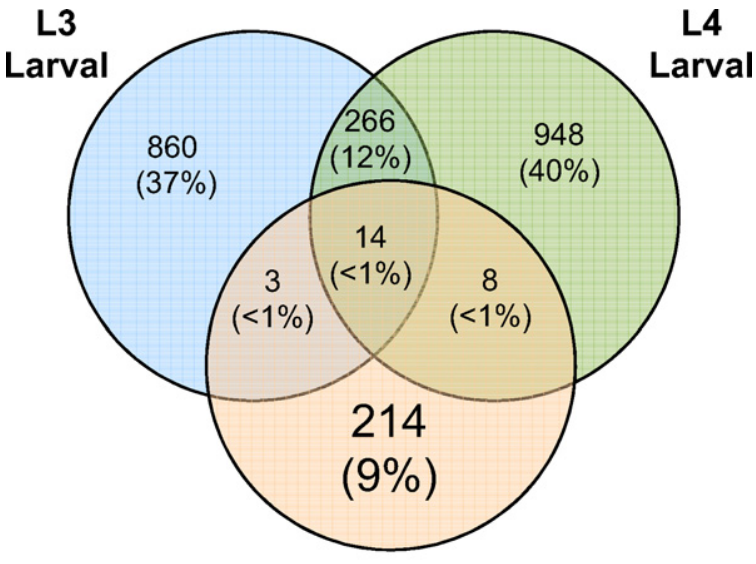

Adult

Fig. 1. Distribution of clusters based on life stages of origin. Total number of clusters is 2284; majority of clusters are represented by only one stage, though further depth of sampling will likely increase representation from multiple stages.

\subsection{Structural and functional annotation, and comparative analysis using bioinformatics approaches}

Transmembrane and signal peptide predictions: Phobius (Kall et al., 2004), a combined Signal Peptide (SP) and Transmembrane (TM) prediction method based on hidden Markov models was used with default settings. Each query sequence was further annotated as SP-only, TM-only or TM with SP. For EST clusters, Phobius annotation was obtained for each contig and then summarized at the NemaGene cluster level (www.nematode.net).

Interpro and Gene Ontology mappings: Default parameters for InterProScan v16.1 were used to search against the InterPro database. Raw InterProScan results for the translated EST contigs were summarized at the EST cluster level. Gene Ontology (GO) annotations were obtained from InterProScan using the goterms flag as a parameter. These annotations were further assigned and displayed graphically by AmiGO utilizing default parameters. Complete GO mappings for the all the genes can be accessed at http://nematode.net/cgi-bin/amigo/go_ostertagia_ostertagi/go.cgi. GO term enrichment was obtained after processing the GO occurrences through the FUNC server (Prufer et al., 2007).

KEGG pathway mappings (Kanehisa and Goto, 2006): WU-BLAST matches of the 0 . ostertagi genes against KEGG database version 46.0 was used for pathway mapping with a filter of $1 \mathrm{e}^{-05}$. Table 3 and supplemental on line ST3 lists mappings for the top scoring and $C$. elegans hits to the KEGG enzymes that meet the $E$-value filter. For hypergeometric comparisons of L3-specific and L4-specific genes, all hits meeting the $E$-value cut-off were used. KEGG associations are available at http://www.nematode.net/ KEGGscan/cgi-bin/KEGGscan_hit_distribution.cgi?species_selection=Ostertagia\%20ostertagi.

\section{Results and discussion}

\subsection{Characteristics of the generated transcripts and the identified genes}

We generated cDNA libraries from three stages of $O$. ostertagi. A total of 7042 ESTs (Table 1) that passed our sequencing and quality control screening were clustered using an internal pipeline (McCarter et al., 2003) to reduce redundancy, and were grouped into 4611 contigs that were translated into amino acid sequences. Valid translations were obtained for 4605 contigs and these clustered into 2284 genes. Gene estimation from ESTs can sometimes result in over-representation because the short transcript length can lead to non-overlapping clusters which are part of a single gene (i.e., fragmentation). Gene fragmentation was estimated at $2.1 \%$ by comparison to $C$. elegans as a reference. The distribution of ESTs per cluster size shows that the largest cluster has 971 ESTs and less than $3 \%$ of clusters have more than 10 ESTs. Sixty-six percent of the clusters contain only one EST. This distribution reflects the abundance of transcripts from the stage-specific expression of the three libraries that were sampled. Highly expressed transcripts are indicative of the expression levels of the stages being sampled and can also represent important functional elements.

Clusters that contain transcripts from all three libraries represent transcripts expressed in all the stages of the parasite and are likely involved in core biological processes. The distribution of the clusters based on library composition is illustrated in Fig. 1. Less than $1 \%$ of all clusters contain transcripts from all stages and 12\% (264) share transcripts among both larval stages. Such low numbers of shared clusters have also been reported in other EST projects (e.g., Mitreva et al., 2004b; Thompson et al., 2005). Proteins enriched in these two specific lifecycle stages are discussed below.

\subsection{Functional classification}

Vast amounts of genomic information are available in public databases and offer a reliable way to identify similarity and/or homology to previously sequenced

Table 1

Ostertagia ostertagi cDNA libraries and properties.

\begin{tabular}{llllr}
\hline Libraries & $\begin{array}{l}\text { Total } \\
\text { ESTs }\end{array}$ & $\begin{array}{l}\text { Total nucleotides } \\
\text { (million bases) }\end{array}$ & $\begin{array}{l}\text { EST mean } \\
\text { length } \pm \text { SD (bases) }\end{array}$ & $\begin{array}{l}\text { Stage-biased } \\
\text { clusters }\end{array}$ \\
\hline L3 & 3207 & 1.41 & $442 \pm 133$ & 1234 \\
L4 & 3582 & 1.38 & $387 \pm 170$ & 1731 \\
Adult & 253 & 0.12 & $509 \pm 202$ & 203 \\
Total & 7042 & 2.91 & $417 \pm 159$ & 342 \\
\hline
\end{tabular}

a 298 clusters comprised of 3689 ESTs originated from 2 or 3 stages were excluded. 


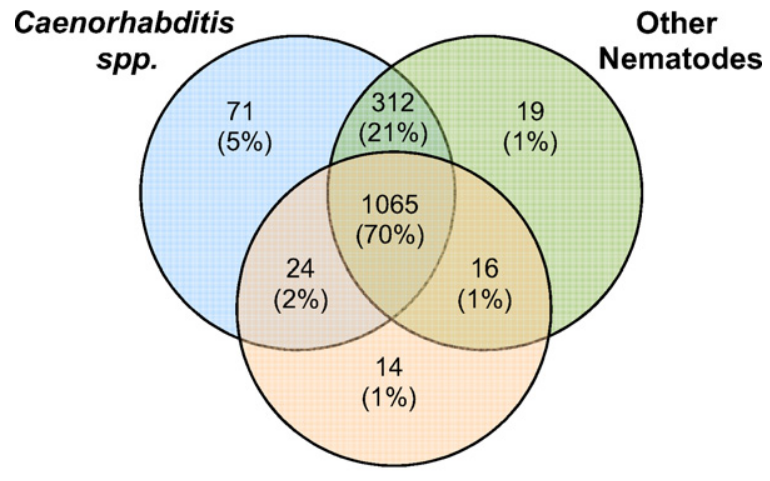

Non Nematodes

Fig. 2. Distribution of $O$. ostertagi cluster BLAST matches by database. Databases used were: Caenorhabditis spp: C. elegans, C. briggsae, $C$. remanei; other nematodes, all GenBank nucleotide data from nematodes except Caenorhabditis species; Non-nematodes, NR with all nematode sequences removed. species. To functionally characterize the 0 . ostertagi genes, three phylogenetic databases were built. The first database includes three Caenorhabditis species (C. elegans, C. briggsae and $C$. remanei); the second database contains all nematode species other than Caenorhabditis; and the last database is the non-redundant protein database from Genbank excluding any nematode sequences (databases built on May 2007). A similarity search of the 2284 genes using WU-BLAST (Gish, 1996-2002) versus these three phylogenetic databases (with $e$-values better than $1 \mathrm{e}^{-05}$ ) indicates that $66 \%(1497 / 2284)$ of the 0 . ostertagi genes have sequence homologs among known and predicted proteins from other species. Over $70 \%$ of these genes matched all three databases (Fig. 2). In addition, the $O$. ostertagi gene clusters were compared to the draft genome of Haemonchus contortus (downloaded on February 10, 2009 from http://www.sanger.ac.uk/Projects/H_contortus/), a clade V Strongylida nematode that infects goats and sheep. This comparison identified 67\% (1529/2284) 0 .

Table 2

The 25 most abundant InterPro domains by 0 . ostertagi cDNA clusters and corresponding mappings in C. elegans.

\begin{tabular}{|c|c|c|c|}
\hline IPR ID & IPR description & O. ostertagi clusters & C. elegans genes \\
\hline IPR008160 & Collagen triple helix repeat & 41 & 197 \\
\hline IPR002486 & Nematode cuticle collagen, N-terminal & 33 & 161 \\
\hline IPR001534 & Transthyretin-like & 21 & 59 \\
\hline IPR013032 & EGF-like region, conserved site & 20 & 241 \\
\hline IPR002048 & Calcium-binding EF-hand & 19 & 166 \\
\hline IPR012336 & Thioredoxin-like fold & 19 & 163 \\
\hline IPR001283 & Allergen V5/Tpx-1 related & 17 & 40 \\
\hline IPR014044 & SCP-like extracellular & 16 & 39 \\
\hline IPR011009 & Protein kinase-like & 16 & 671 \\
\hline IPR011992 & EF-Hand type & 16 & 123 \\
\hline IPR003006 & Immunoglobulin & 15 & 27 \\
\hline IPR012335 & Thioredoxin fold & 15 & 142 \\
\hline IPR003582 & Metridin-like ShK toxin & 15 & 125 \\
\hline IPR000583 & Glutamine amidotransferase, class-II & 14 & 12 \\
\hline IPR000719 & Protein kinase, core & 14 & 611 \\
\hline IPR012677 & Nucleotide-binding, alpha-beta plait & 14 & 154 \\
\hline IPR010916 & TonB box, conserved site & 12 & 25 \\
\hline IPR007087 & Zinc finger, C2H2-type & 12 & 262 \\
\hline IPR000504 & RNA recognition motif, RNP-1 & 12 & 159 \\
\hline IPR008978 & HSP20-like chaperone & 12 & 27 \\
\hline IPR002068 & Heat shock protein Hsp20 & 12 & 22 \\
\hline IPR010920 & Like-Sm ribonucleoprotein-related & 11 & 19 \\
\hline IPR001806 & Ras GTPase & 11 & 90 \\
\hline IPR001436 & Alpha crystallin/Heat shock protein & 11 & 21 \\
\hline IPR001163 & Like-Sm ribonucleoprotein, core & 11 & 18 \\
\hline
\end{tabular}

Table 3

KEGG metabolic pathways mapped by 0 . ostertagi cDNA clusters and corresponding mappings in C. elegans.

\begin{tabular}{|c|c|c|c|c|}
\hline \multirow[t]{2}{*}{ KEGG pathways } & \multicolumn{2}{|c|}{ O. ostertagi } & \multicolumn{2}{|c|}{ C. elegans } \\
\hline & Genes & Enzymes & Genes & Enzymes \\
\hline 1. Metabolism & 352 & 174 & 4494 & 642 \\
\hline 1.1 Carbohydrate metabolism & 107 & 61 & 1432 & 171 \\
\hline 1.2 Energy metabolism & 91 & 23 & 450 & 51 \\
\hline 1.3 Lipid metabolism & 76 & 43 & 1219 & 134 \\
\hline 1.4 Nucleotide metabolism & 62 & 24 & 1015 & 78 \\
\hline 1.5 Amino acid metabolism & 72 & 48 & 1040 & 195 \\
\hline 1.6 Metabolism of other amino acids & 17 & 14 & 319 & 52 \\
\hline 1.7 Glycan biosynthesis and metabolism & 35 & 16 & 678 & 72 \\
\hline 1.8 Biosynthesis of polyketides and nonribosomal peptides & 9 & 7 & 197 & 12 \\
\hline 1.9 Metabolism of cofactors and vitamins & 82 & 30 & 843 & 98 \\
\hline 1.10 Biosynthesis of secondary metabolites & 34 & 18 & 661 & 47 \\
\hline 1.11 Xenobiotics biodegradation and metabolism & 50 & 22 & 806 & 52 \\
\hline
\end{tabular}


ostertagi genes that had homologies with $e$-values better than $1 \mathrm{e}^{-05}$, of which 148 were homologies not shared with the three phylogenetic databases presented in Fig. 2. The average identity between these genes was $70 \%$, with one third having identities greater than $80 \%$ (data not shown). In all probability, these shared gene products are widely conserved among metazoans and associated with core biological processes. Five percent of Ostertagia genes with identity only to genes from Caenorhabditis species are potential nematode Clade $\mathrm{V}$ lineage-specific genes whereas the 639 O. ostertagi genes that did not share homology with any database are likely Trichostrongyloidea or Ostertagia-specific sequences.
Comparison to C. elegans showed that 52\% (1187/2284) of the $O$. ostertagi genes have well-conserved putative orthologs in C. elegans. These orthologs were determined based on C. elegans and O. ostertagi gene pairs that have the highest sequence similarity to each other in reciprocal WU-BLAST searches (with $e$-values better than $1 \mathrm{e}^{-05}$ ). Gene groups that had a high degree of conservation (with $e$-value better than $1 \mathrm{e}^{-100}$ ) included ribosomal proteins, collagen proteins, cytochrome subunits, zinc metalloproteases and serine/threonine kinases (Supplemental Information, ST1). RNA interference (RNAi) has been reported in 0 . ostertagi in a few genes but knockdown effects have not been reliable (Visser et al., 2006). However, extrapolation

(A) Biological Process

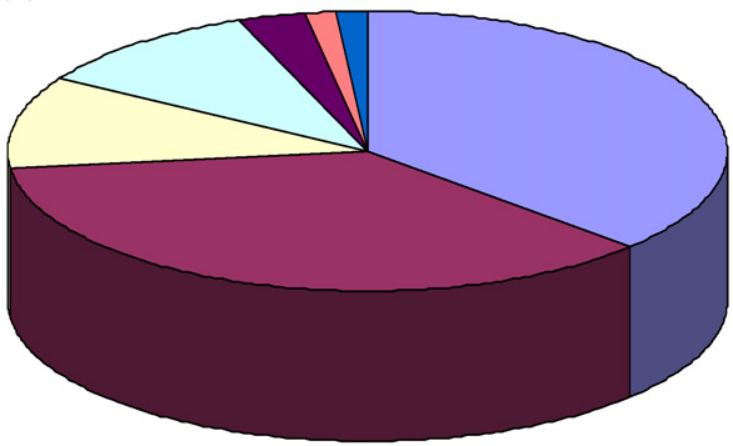

$\square$ cellular process

metabolic process

Ilocalization

Destablishment of localization

biological regulation

$\square$ developmental process

$\square$ others

(B)

Cellular Component

$\square$ cell

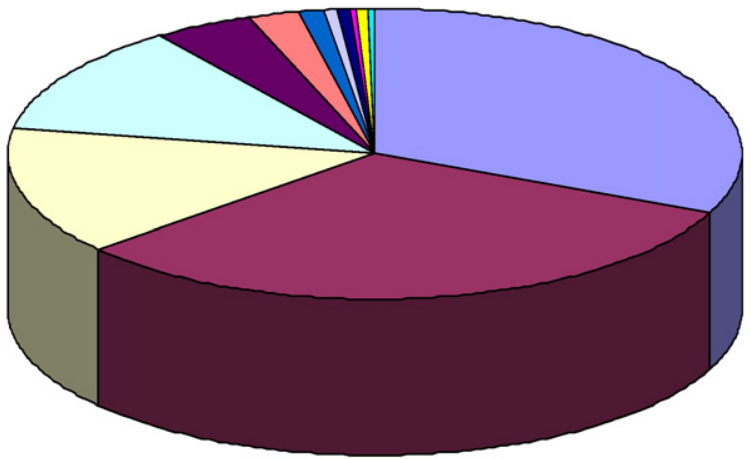

$\square$ cell part

$\square$ organelle

$\square$ macromolecular complex

organelle part

$\square$ extracellular region

envelope

$\square$ extracellular matrix

extracellular region part

$\checkmark$ synapse part

$\square$ synapse

$\square$ others

(C)

Molecular Function
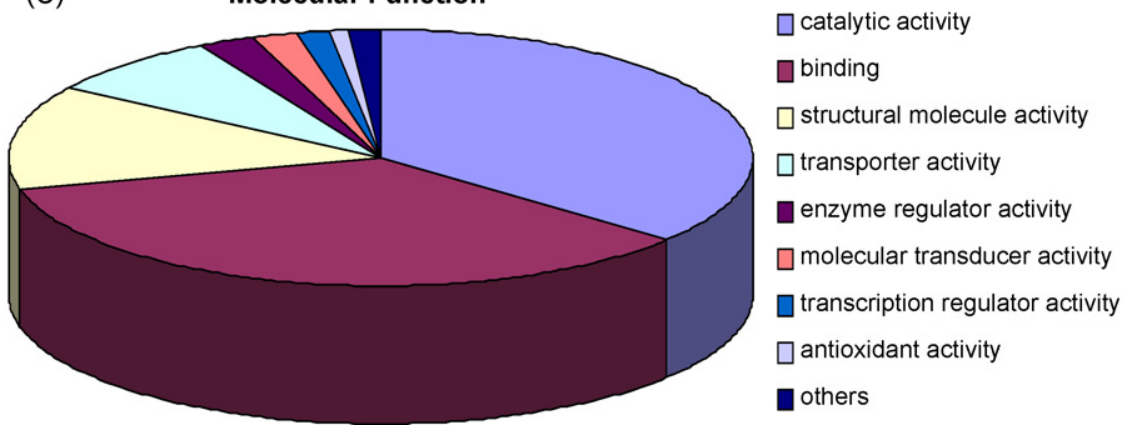

Fig. 3. Percentage representation of Gene Ontology (GO) categories for O. ostertagi clusters: (A) biological processes; (B) cellular component; (C) molecular function. 
from the C. elegans orthologs/homologs with observed RNAi phenotypes can be highly informative for functional analysis. Of orthologous genes, $46 \%$ have observable $C$. elegans RNAi phenotypes. Further, when phenotypes were observed, $82 \%$ exhibited severe effects including embryonic, larval, or adult lethal, sterile adults or progeny, and larval or adult growth arrest (Supplemental Information, ST2).

The InterPro (Apweiler et al., 2001) database was searched to identify similarities to annotated protein domains and 1172 genes shared sequence similarity with 1035 unique Interpro domains (Supplemental Information, ST4). Well-represented domains include collagen triple helix repeat, nematode cuticle collagen, EGF-like region and transthyretin-like (Table 2). Among the sequences evaluated, 884 genes have GO (The Gene Ontology, 2008) mappings and the three GO categories, biological process (670 genes), cellular component (451 genes) and molecular function (764 genes). Data constituting these mappings are shown in Fig. 3.

Unique clusters (605) mapped to 143 biochemical pathways that are grouped in 11 KEGG categories (Kanehisa and Goto, 2006) (Table 3). A complete listing of these pathways is available as supplementary material on line (Supplemental Information ST3) and graphical representations of the KEGG mappings are available at www.nematode.net (Martin et al., 2009). This viewer provides associations to specific enzyme commission (EC) numbers, KEGG Orthology identifiers, significance of the similarity and the $O$. ostertagi genes associated with the enzyme. Using this web tool, it is possible to make comparisons of the mappings between $O$. ostertagi and another nematode species for any enzyme in the metabolic pathways. This visualization can shed insight into the differences in parasitism, adaptations to the environment, etc., between the 32 partial nematode genomes available currently. Comparisons with the $C$. elegans KEGG viewer provides a mechanism to associate RNAi phenotype information assigned to the $C$. elegans genes associated with the pathways. Potential chokepoints in these metabolic pathways can be determined and this enables the identification of genes of interest based on their function.

Most nematode vaccine targets are excretory/secretory (ES) products or intestinal antigens that are membranebound. Excretory-secretory protein products are often involved in host-parasite interactions or have a role in parasite development and survival within the host. As such, they present suitable targets for alternative intervention strategies (Newton and Meeusen, 2003). However, it should be noted that prediction of signal peptides defines cellular events and not parasitological events. Therefore, a "secretory signal" characterizes the possibility that the protein in question will be secreted from the cell and not necessarily from the parasite and into the host. Comparing the hidden Markov models of different sequenced regions, we identified 2540 . ostertagi genes that have signal peptides for secretion, 355 genes with transmembrane domains and 44 genes with both features. Of the genes with SP, 43\% had associations to 122 Interpro domains and $49 \%$ of the TM to 187 domains (Supplemental Information, Table ST5).
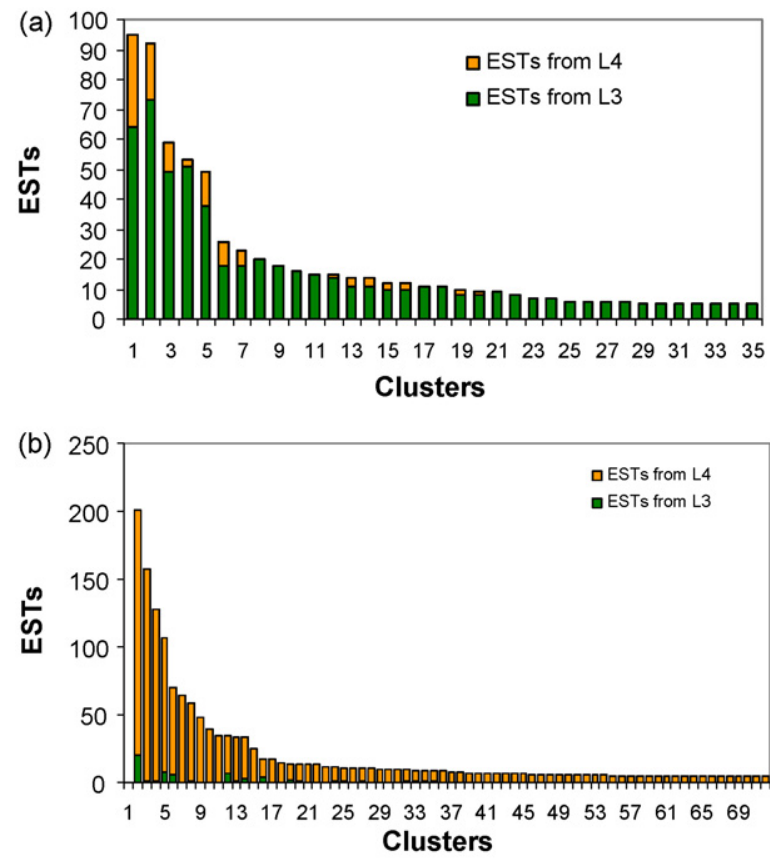

Fig. 4. Cluster composition of stage-specific clusters: (A) EST distribution in L3-specific transcripts (for better visualization the biggest cluster is excluded); (B) EST distribution in L4-specific clusters.

\subsection{Stage-enriched expression}

\subsubsection{Stage-biased expression}

Ostertagia L3- and L4-biased and -specific clusters were identified, functionally characterized and compared to provide insight into adaptation and enrichment in each stage. The stage-biased clusters, comprised entirely of one of the two stages, resulted in 870 L3- and 955 L4-biased genes. Using a hypergeometric distribution analysis on KEGG metabolic pathways, we identified the pathways enriched in each of the stages. Fatty acid metabolism, metabolism of xenobiotics by cytochrome P450, C21steroid hormone metabolism, linoleic acid metabolism, arachidonic acid metabolism, galactose metabolism and pentose and glucuronate interconversions are among the most enriched pathways among genes biased to L3. Pathways enriched in L4 stage include oxidative phosporylation and ubiquinone biosynthesis. Fatty acids have a role in locomotion and infection of host of L3 larvae in other nematodes (Medica and Sukhdeo, 1997) and xenobiotic metabolism in nematodes has relevance for anthelminthic drug applications (Kotze et al., 2006). The enriched pathways from both stages are listed in Supplemental Information (ST6).

Analysis of GO terms using the FUNC web server (Prufer et al., 2007) with this dataset show that in the L3 stage, oxidoreductase activity, signal transduction, binding, aromatic compound biosynthesis and GTP-cyclohydrolase I activity are significantly over-represented. Genes involved in the degradation of fatty acids and aromatic compounds show up-regulation in the $C$. elegans dauer stage (Burnell et al., 2005). Phosphate transport, structural constituents of the cuticle, serine-type peptidase activity, 
Table 4

Transcripts significantly enriched in L3 larval.

\begin{tabular}{|c|c|c|c|c|c|c|c|c|c|}
\hline \multirow[t]{2}{*}{ Genes } & \multirow[t]{2}{*}{ L3 ESTs } & \multirow[t]{2}{*}{ L4 ESTs } & \multirow[t]{2}{*}{ Contigs } & \multicolumn{3}{|l|}{ Best NR hit } & \multicolumn{3}{|c|}{$\begin{array}{l}\text { Signal peptide and transmem- } \\
\text { brane domain }\end{array}$} \\
\hline & & & & Acc\# & Description & $E$-value range & SP & TM & SP and TM \\
\hline OS00004.cl & 963 & 8 & 163 & AAC06296.1 & Ostertagia ostertagi GTP-cyclohydrolase & $2.4 \mathrm{e}-102-5.6 \mathrm{e}-16$ & - & - & - \\
\hline OS00029.cl & 18 & 5 & 19 & NP_497786.2 & $\begin{array}{l}\text { Caenorhabditis elegans MethylMalonylCoA } \\
\text { Mutase homolog family member }\end{array}$ & $4.4 \mathrm{e}-87-1.4 \mathrm{e}-15$ & - & - & - \\
\hline OS00996.cl & 5 & 0 & 1 & NP_502959.1 & $\begin{array}{l}\text { Caenorhabditis elegans RHO (small G protein) } \\
\text { family member (rho-1) }\end{array}$ & $4.2 \mathrm{e}-98-4.2 \mathrm{e}-98$ & - & - & \\
\hline OS01074.cl & 10 & 2 & 2 & NP_509853.2 & $\begin{array}{l}\text { Caenorhabditis elegans AdaPTin or adaptin-related } \\
\text { protein family member (apt-9) }\end{array}$ & $6.5 e-06-6.5 e-06$ & - & - & - \\
\hline OS00821.cl & 6 & 0 & 2 & NP_509136.1 & $\begin{array}{l}\text { Caenorhabditis elegans MAD-Like family member } \\
\text { (mdl-1) }\end{array}$ & $2.5 e-36-2.1 e-32$ & - & - & - \\
\hline OS00054.cl & 18 & 0 & 8 & NP_579949.2 & Ancylostoma duodenale CYTB_15956 cytochrome b & $7.6 e-99-4.6 e-76$ & - & 8 & - \\
\hline OS00041.cl & 73 & 19 & 63 & BAE72489.1 & Cooperia oncophora cytochrome oxidase subunit 1 & $2.5 e-107-5.6 e-71$ & 1 & 57 & 5 \\
\hline OS00039.cl & 51 & 2 & 16 & САH 23215.2 & Ostertagia ostertagi cytochrome-c oxidase subunit II & $4.6 e-108-1.0 e-41$ & - & 15 & - \\
\hline OS00511.cl & 8 & 2 & 8 & NP_851327.1 & Cooperia oncophora NADH dehydrogenase subunit 2 & $2.3 e-65-6.9 e-34$ & - & 8 & - \\
\hline OS00515.cl & 10 & 2 & 4 & AAX24133.1 & Onchocerca volvulus alpha-tubulin & $8.2 e-26-8.2 e-26$ & - & 1 & - \\
\hline OS00408.cl & 11 & 3 & 7 & AAR85527.1 & Meloidogyne incognita $14-3-3 \mathrm{~b}$ protein & $3.8 \mathrm{e}-113-1.1 \mathrm{e}-28$ & - & - & - \\
\hline OS00007.cl & 18 & 8 & 21 & Q17761 & $\begin{array}{l}\text { Caenorhabditis elegans 6-phosphogluconate } \\
\text { dehydrogenase, decarboxylating }\end{array}$ & $4.8 \mathrm{e}-74-1.8 \mathrm{e}-13$ & - & 1 & - \\
\hline OS00049.cl & 64 & 31 & 13 & ABX79385.1 & $\begin{array}{l}\text { Dictyocaulus viviparus glyceraldehyde-3-phosphate } \\
\text { dehydrogenase }\end{array}$ & $1.2 e-146-2.7 e-23$ & - & - & - \\
\hline OS01410.cl & 11 & 3 & 14 & NP_001022079.1 & $\begin{array}{l}\text { Caenorhabditis elegans Aldehyde deHydrogenase } \\
\text { family member (alh-8) }\end{array}$ & $2.0 \mathrm{e}-20-7.4 \mathrm{e}-14$ & 3 & - & - \\
\hline OS00009.cl & 15 & 0 & 11 & Q95XM2 & $\begin{array}{l}\text { Caenorhabditis elegans probable peptidylglycine } \\
\text { alpha-hydroxylating monooxygenase } \\
\text { Y71G12B.4 precursor (PHM) }\end{array}$ & $1.2 \mathrm{e}-63-4.0 \mathrm{e}-38$ & 10 & - & - \\
\hline OS00030.cl & 6 & 0 & 3 & NP_500410.2 & $\begin{array}{l}\text { Caenorhabditis elegans UDP-GlucuronosylTransferase } \\
\text { family member (ugt- } 45 \text { ) }\end{array}$ & $1.1 \mathrm{e}-42-1.1 \mathrm{e}-42$ & - & - & - \\
\hline OS00122.cl & 38 & 11 & 4 & NP_001041224.1 & Caenorhabditis elegans hypothetical protein & $8.9 e-18-1.2 e-15$ & - & 4 & - \\
\hline OS01031.cl & 8 & 1 & 3 & NP_494448.1 & Caenorhabditis elegans hypothetical protein & $5.0 e-63-2.0 e-50$ & - & - & - \\
\hline OS00407.cl & 9 & 0 & 5 & NP_505575.2 & Caenorhabditis elegans hypothetical protein & $1.7 e-11-4.7 e-11$ & - & - & - \\
\hline OS00389.cl & 11 & 0 & 10 & AAC06294.1 & Ostertagia ostertagi hypothetical protein & $3.7 e-35-7.3 e-23$ & - & 5 & 5 \\
\hline OS00975.cl & 5 & 0 & 1 & NP_500023.1 & Caenorhabditis elegans hypothetical protein & $7.1 \mathrm{e}-48-7.1 \mathrm{e}-48$ & - & - & - \\
\hline OS00038.cl & 11 & 0 & 6 & CAN86922.1 & Caenorhabditis elegans hypothetical protein & $2.6 e-57-2.0 e-50$ & - & 6 & - \\
\hline OS00125.cl & 5 & 0 & 4 & АAT02162.1 & Dictyocaulus viviparus hypothetical protein & $1.4 \mathrm{e}-67-7.1 \mathrm{e}-48$ & 4 & - & - \\
\hline OS00119.cl & 7 & 0 & 6 & YP_001230869.1 & Geobacter uraniumreducens hypothetical protein & $4.5 e-07-4.5 e-07$ & 6 & - & - \\
\hline OS00050.cl & 16 & 0 & 14 & CAE73111.1 & Caenorhabditis briggsae hypothetical protein & $1.7 e-69-1.6 e-25$ & 2 & - & - \\
\hline OS00001.cl & 5 & 0 & 4 & XP_001675620.1 & Caenorhabditis briggsae AF16 hypothetical protein & $2.5 e-61-7.7 e-51$ & 4 & - & - \\
\hline OS00080.cl & 5 & 0 & 5 & XP_001671626.1 & Caenorhabditis briggsae AF16 hypothetical protein & $1.7 e-76-2.0 e-52$ & - & - & - \\
\hline OS00013.cl & 49 & 10 & 23 & CAE73030.1 & Caenorhabditis briggsae hypothetical protein & $2.8 e-60-1.3 e-10$ & - & - & - \\
\hline OS00002.cl & 6 & 0 & 5 & NP_505353.1 & Caenorhabditis elegans hypothetical protein & $1.7 e-21-7.7 e-19$ & - & - & - \\
\hline OS00797.cl & 7 & 0 & 7 & AAC08432.1 & Ostertagia ostertagi unknown protein & $1.2 \mathrm{e}-27-8.6 \mathrm{e}-20$ & - & - & - \\
\hline OS01519.cl & 14 & 1 & 15 & AAC08346.1 & Ostertagia ostertagi unknown protein & $2.5 e-20-3.6 e-14$ & 8 & - & - \\
\hline OS00477.cl & 5 & 0 & 5 & AAC08429.1 & Ostertagia ostertagi unknown protein & $5.6 e-32-2.3 e-19$ & 1 & - & - \\
\hline OS00405.cl & 8 & 0 & 3 & - & Novel & - & - & - & - \\
\hline OS00024.cl & 20 & 0 & 15 & - & Novel & - & - & 2 & - \\
\hline OS00062.cl & 5 & 0 & 3 & - & Novel & - & - & 1 & - \\
\hline OS04517.cl & 6 & 0 & 6 & - & Novel & - & 2 & - & - \\
\hline
\end{tabular}


Transcripts significantly enriched in L4 larval.

\begin{tabular}{|c|c|c|c|c|c|c|c|c|c|}
\hline \multirow[t]{2}{*}{ Genes } & \multirow[t]{2}{*}{ L3 ESTs } & \multirow[t]{2}{*}{ L4 ESTs } & \multirow[t]{2}{*}{ Contigs } & \multicolumn{3}{|l|}{ Best NR Hit } & \multicolumn{3}{|c|}{$\begin{array}{l}\text { Signal Peptide and Transmem- } \\
\text { brane Domain }\end{array}$} \\
\hline & & & & Acc\# & Description & $E$-value range & SP & TM & SP \& TM \\
\hline OS00943.cl & 1 & 8 & 3 & NP_740944.1 & $\begin{array}{l}\text { Caenorhabditis elegans ribosomal protein, small } \\
\text { subunit family member (rps-20) }\end{array}$ & $7.1 \mathrm{e}-48-2.9 \mathrm{e}-42$ & - & - & - \\
\hline OS00881.cl & 1 & 8 & 7 & ABP52062.1 & Ancylostoma duodenale ribosomal protein S27e & $7.8 e-42-6.9 e-34$ & 1 & - & - \\
\hline OS00307.cl & 1 & 8 & 7 & NP_497721.1 & $\begin{array}{l}\text { Caenorhabditis elegans ribosomal protein, large } \\
\text { subunit family member (rpl-16) }\end{array}$ & $2.2 e-78-2.8 e-69$ & - & - & - \\
\hline OS00885.cl & 1 & 10 & 3 & XP_001671152.1 & Caenorhabditis briggsae hypothetical protein & $1.2 e-36-3.3 e-27$ & - & - & - \\
\hline OS00344.cl & 2 & 11 & 3 & XP_001668382.1 & Caenorhabditis briggsae hypothetical protein & $4.2 \mathrm{e}-50-2.5 \mathrm{e}-22$ & - & - & - \\
\hline OS00701.cl & 4 & 13 & 5 & XP_001666264.1 & Caenorhabditis briggsae hypothetical protein & $2.9 e-35-6.8 e-27$ & - & - & - \\
\hline OS00233.cl & 1 & 12 & 12 & XP_001669407.1 & Caenorhabditis briggsae hypothetical protein & $4.1 \mathrm{e}-45-3.1 \mathrm{e}-08$ & - & - & - \\
\hline OS00785.cl & 0 & 5 & 2 & XP_001665645.1 & Caenorhabditis briggsae hypothetical protein & $4.2 \mathrm{e}-91-4.2 \mathrm{e}-91$ & - & - & - \\
\hline OS00944.cl & 0 & 5 & 3 & XP_001665512.1 & Caenorhabditis briggsae hypothetical protein & $5.7 e-78-1.9 e-38$ & - & - & - \\
\hline OS01019.cl & 0 & 7 & 2 & NP_498300.2 & $\begin{array}{l}\text { Caenorhabditis elegans muscle LIM protein/ } \\
\text { cysteine-rich protein family member (mlp-1) }\end{array}$ & $2.5 e-52-1.8 e-33$ & - & - & - \\
\hline OS00224.cl & 0 & 5 & 3 & NP_495170.1 & $\begin{array}{l}\text { Caenorhabditis elegans temporarily assigned gene } \\
\text { name family member (tag-234) }\end{array}$ & $1.3 e-09-1.3 e-09$ & - & 2 & 1 \\
\hline OS00468.cl & 0 & 6 & 3 & CAD22110.1 & Ostertagia ostertagi keratin & $1.1 \mathrm{e}-67-1.1 \mathrm{e}-65$ & 1 & - & - \\
\hline OS00687.cl & 1 & 33 & 7 & CAC44258.1 & Ostertagia ostertagi $17 \mathrm{kDa}$ ES antigen protein & $1.0 \mathrm{e}-55-1.1 \mathrm{e}-44$ & - & - & - \\
\hline OS00999.cl & 0 & 10 & 5 & NP_491501.1 & $\begin{array}{l}\text { Caenorhabditis elegans paralyzed arrest at two-fold } \\
\text { family member (pat-10) }\end{array}$ & $2.2 e-76-4.3 e-18$ & - & - & - \\
\hline OS00941.cl & 0 & 5 & 2 & XP_001666514.1 & Caenorhabditis briggsae hypothetical protein & $6.7 e-36-1.4 e-17$ & - & - & - \\
\hline OS01020.cl & 0 & 64 & 8 & NP_500520.1 & $\begin{array}{l}\text { Caenorhabditis elegans COLlagen family member } \\
\text { (col-34) }\end{array}$ & $1.7 e-06-1.7 e-06$ & - & 2 & - \\
\hline OS00275.cl & 0 & 10 & 4 & XP_001667179.1 & Caenorhabditis briggsae hypothetical protein & $9.4 e-46-6.4 e-31$ & - & - & - \\
\hline OS00677.cl & 3 & 31 & 7 & |XP_001670744.1 & Caenorhabditis briggsae hypothetical protein & $9.5 e-53-1.4 e-26$ & - & - & - \\
\hline OS00932.cl & 0 & 5 & 2 & ААВ37842.2 & Caenorhabditis elegans collagen protein 20 & $7.2 e-55-9.9 e-19$ & - & 2 & - \\
\hline OS00291.cl & 0 & 9 & 9 & XP_001670744.1 & Caenorhabditis briggsae hypothetical protein & $3.7 e-51-1.3 e-41$ & - & 3 & - \\
\hline OS00357.cl & 0 & 6 & 2 & AAB69960.1 & Caenorhabditis elegans collagen protein 141 & $1.3 e-23-1.3 e-23$ & - & 1 & - \\
\hline OS00685.cl & 0 & 35 & 32 & XP_001668726.1 & Caenorhabditis briggsae hypothetical protein & $2.0 \mathrm{e}-27-3.1 \mathrm{e}-22$ & - & - & - \\
\hline OS00931.cl & 0 & 5 & 2 & XP_001671845.1 & Caenorhabditis briggsae hypothetical protein & $2.4 e-72-2.4 e-72$ & - & 2 & - \\
\hline OS00230.cl & 0 & 25 & 13 & XP_001666450.1 & Caenorhabditis briggsae hypothetical protein & $1.8 e-65-2.5 e-52$ & - & 2 & - \\
\hline OS00697.cl & 0 & 5 & 2 & NP_506053.1 & Caenorhabditis elegans hypothetical protein & $1.8 \mathrm{e}-19-1.8 \mathrm{e}-19$ & - & - & - \\
\hline OS00052.cl & 8 & 99 & 31 & XP_001892268.1 & $\begin{array}{l}\text { Brugia malayi nematode cuticle collagen } \\
\mathrm{N} \text {-terminal domain containing protein }\end{array}$ & $2.2 \mathrm{e}-21-2.2 \mathrm{e}-21$ & 2 & 26 & - \\
\hline OS00242.cl & 0 & 5 & 5 & XP_001679049.1 & Caenorhabditis briggsae hypothetical protein & $3.9 e-56-3.0 e-10$ & - & - & - \\
\hline OS00867.cl & 0 & 11 & 9 & 097391 & $\begin{array}{l}\text { Trichostrongylus colubriformis } 30 \mathrm{kDa} \text { antigenic } \\
\text { glycoprotein precursor (ESGP30) }\end{array}$ & $1.8 \mathrm{e}-17-5.4 \mathrm{e}-11$ & 3 & 2 & - \\
\hline OS01046.cl & 7 & 28 & 29 & NP_506707.2 & $\begin{array}{l}\text { Caenorhabditis elegans RAP homolog (vertebrate } \\
\text { Rap GTPase family) family member (rap-2) }\end{array}$ & $2.1 \mathrm{e}-64-4.5 \mathrm{e}-23$ & - & 5 & - \\
\hline OS00282.cl & 1 & 10 & 7 & NP_499536.1 & $\begin{array}{l}\text { Caenorhabditis elegans MIF (macrophage migration } \\
\text { inhibitory factor) related family member (mif- } 1 \text { ) }\end{array}$ & $2.4 \mathrm{e}-24-1.4 \mathrm{e}-21$ & - & - & - \\
\hline OS03801.cl & 0 & 5 & 5 & NP_496707.1 & Caenorhabditis elegans hypothetical protein & $7.5 e-21-6.4 e-15$ & - & 2 & - \\
\hline OS00862.cl & 1 & 58 & 7 & XP_001668977.1 & Caenorhabditis briggsae hypothetical protein & $8.8 e-82-7.8 e-58$ & - & - & - \\
\hline OS00267.cl & 0 & 10 & 7 & CAL85590.1 & Drosophila simulans Fst & $7.1 \mathrm{e}-08-7.1 \mathrm{e}-08$ & - & - & - \\
\hline OS00630.cl & 0 & 13 & 2 & XP_001716140.1 & Homo sapiens hypothetical protein, partial & $4.5 e-14-1.5 e-13$ & - & - & - \\
\hline OS00223.cl & 0 & 8 & 8 & CAB41740.1 & Litomosoides sigmodontis secretory protein (LS110p) & $9.8 e-12-9.8 e-12$ & 1 & - & - \\
\hline OS01023.cl & 0 & 7 & 2 & CAE56607.1 & Caenorhabditis briggsae hypothetical protein & $1.9 e-77-1.9 e-77$ & - & - & - \\
\hline
\end{tabular}




\begin{tabular}{|c|c|c|c|c|c|c|c|c|c|}
\hline OS00198.cl & 0 & 14 & 7 & AAD31839.1 & $\begin{array}{l}\text { Ancylostoma caninum ancylostoma-secreted } \\
\text { protein } 1 \text { precursor }\end{array}$ & $9.0 e-24-1.3 e-20$ & 2 & - & - \\
\hline OS00977.cl & 0 & 5 & - & & - & - & 1 & - & - \\
\hline OS00966.cl & 0 & 5 & 1 & NP_496755.1 & Caenorhabditis elegans hypothetical protein & $9.9 e-19-9.9 e-19$ & - & - & - \\
\hline OS00668.cl & 0 & 5 & 4 & CAG25499.1 & Ostertagia ostertagi heat shock protein 20 & $1.2 \mathrm{e}-36-1.2 \mathrm{e}-36$ & - & - & - \\
\hline OS00256.cl & 0 & 5 & 3 & Q22700 & Caenorhabditis elegans membrane protein & $9.9 e-19-2.5 e-13$ & - & 3 & - \\
\hline OS00618.cl & 0 & 6 & 5 & ZP_01509120.1 & Burkholderia phytofirmans PE-PGRS family protein & $3.1 \mathrm{e}-09-3.1 \mathrm{e}-09$ & 3 & 2 & - \\
\hline OS00180.cl & 0 & 39 & 26 & CAD20463.1 & Ostertagia ostertagi globin-like ES protein F6 & $8.3 e-70-4.9 e-49$ & 3 & - & - \\
\hline OS00159.cl & 0 & 7 & 4 & CAE12199.1 & Haemonchus contortus aspartyl protease precursor & $4.9 e-56-1.6 e-43$ & - & - & - \\
\hline OS02535.cl & 0 & 12 & 12 & XP_850042.1 & $\begin{array}{l}\text { Canis familiaris similar to eukaryotic translation } \\
\text { elongation factor } 1 \text { alpha } 2\end{array}$ & $2.6 \mathrm{e}-11-2.6 \mathrm{e}-11$ & - & - & - \\
\hline OS00268.cl & 0 & 17 & 12 & NP_502894.1 & Caenorhabditis elegans hypothetical protein & $3.1 \mathrm{e}-08-1.3 \mathrm{e}-07$ & 1 & _- & - \\
\hline OS01546.cl & 20 & 181 & 201 & CAC17797.1 & Ostertagia ostertagi SXC1 protein & $2.8 e-14-9.7 e-12$ & - & - & - \\
\hline OS02354.cl & 0 & 13 & 13 & CAD20812.1 & Ostertagia ostertagi SXC2 protein & $1.6 \mathrm{e}-18-6.2 \mathrm{e}-17$ & - & - & - \\
\hline OS00678.cl & 0 & 5 & 3 & NP_494678.2 & Caenorhabditis elegans hypothetical protein & $5.7 e-14-3.2 e-13$ & - & - & - \\
\hline OS00235.cl & 0 & 8 & 7 & XP_001677820.1 & Caenorhabditis briggsae hypothetical protein & $3.5 \mathrm{e}-30-1.4 \mathrm{e}-26$ & - & - & - \\
\hline OS00716.cl & 0 & 5 & 4 & XP_001669609.1 & Caenorhabditis briggsae hypothetical protein & $1.2 e-18-1.3 e-13$ & - & - & - \\
\hline OS00897.cl & 0 & 6 & 4 & XP_001668233.1 & Caenorhabditis briggsae hypothetical protein & $2.3 e-26-4.7 e-19$ & - & - & - \\
\hline OS00260.cl & 0 & 5 & 5 & NP_498341.1 & Caenorhabditis elegans hypothetical protein & $2.9 e-42-2.9 e-42$ & 1 & - & - \\
\hline OS02221.cl & 1 & 9 & 10 & NP_493074.2 & Caenorhabditis elegans hypothetical protein & $5.1 \mathrm{e}-38-5.1 \mathrm{e}-38$ & - & 1 & - \\
\hline OS02313.cl & 1 & 127 & 128 & ZP_01774245.1 & $\begin{array}{l}\text { Geobacter bemidjiensis Bem conserved } \\
\text { hypothetical protein }\end{array}$ & $2.5 e-21-2.5 e-21$ & - & 39 & - \\
\hline OS00355.cl & 6 & 64 & 70 & XP_001668280.1 & Caenorhabditis briggsae hypothetical protein & $2.9 \mathrm{e}-44-5.2 \mathrm{e}-13$ & - & - & - \\
\hline OS00157.cl & 1 & 11 & 8 & NP_495810.1 & Caenorhabditis elegans hypothetical protein & $2.2 \mathrm{e}-62-1.1 \mathrm{e}-40$ & - & 3 & - \\
\hline OS00175.cl & 0 & 7 & 5 & CAE71812.1 & Caenorhabditis briggsae hypothetical protein & $1.8 \mathrm{e}-28-2.7 \mathrm{e}-25$ & - & - & - \\
\hline OS00726.cl & 0 & 6 & 5 & NP_501158.1 & Caenorhabditis elegans hypothetical protein & $1.9 \mathrm{e}-06-1.9 \mathrm{e}-06$ & - & - & - \\
\hline OS03722.cl & 0 & 7 & - & - & Novel & - & - & 2 & - \\
\hline OS01053.cl & 1 & 157 & - & - & Novel & - & - & 4 & - \\
\hline OS02387.cl & 0 & 48 & - & - & Novel & - & - & - & - \\
\hline OS02814.cl & 0 & 7 & - & - & Novel & - & - & - & - \\
\hline OS00859.cl & 0 & 11 & - & _- & Novel & _ & _ & - & - \\
\hline OS00187.cl & 0 & 7 & - & - & Novel & - & - & 3 & - \\
\hline OS00635.cl & 0 & 6 & - & - & Novel & - & _- & - & - \\
\hline OS02629.cl & 0 & 6 & - & - & Novel & - & - & - & - \\
\hline OS03396.cl & 0 & 5 & - & - & Novel & - & - & - & - \\
\hline OS00957.cl & 0 & 5 & - & - & Novel & _- & - & - & - \\
\hline OS02432.cl & 0 & 6 & - & - & Novel & - & - & - & - \\
\hline OS00312.cl & 0 & 6 & - & - & Novel & - & - & - & - \\
\hline
\end{tabular}


intracellular part and extracellular region are enriched in the L4 genes. A list of over-represented GO terms is presented in Supplemental Information (ST7). Enzymes belonging to the enriched KEGG pathways and functional domains of these GO terms reveal functional profiles that illustrate stage-specific adaptation and therefore, can serve as potential focus points for designing control studies.

\subsubsection{Stage-specific expression}

The stage-specific clusters were identified based on stage of origin and published statistical methodology (Audic and Claverie, 1997). While some of these will not remain stagespecific once more sequencing is performed, at present we were able to identify 36 possible L3-specific genes (comprised of 1527 ESTs) and 71 L4-specific genes (1428 ESTs) (significance threshold of 0.05) and the EST composition of these clusters are shown in Fig. 4. Investigating the differences in function between these two sets of genes provided valuable insight into the parasite's life-cycle and can help identifying putative targets for designing effective vaccines and drugs targeting specific stages.

WU-BLAST analysis of the 36 L3-specific clusters shows that the majority of them have similarity to known or hypothetical proteins (Table 4). The most well-represented functional category is the GTP-cyclohydrolase enzyme. This transcript, represented by 971 ESTs, putatively encodes GTP-cyclohydrolase I (EC 3.5.4.1.6) enzyme which is part of the KEGG folate biosynthesis pathway. This enzyme has previously been studied using RT-PCR and shown to be up-regulated in the L3 compared to other stages and its expression is localized to muscle and intestinal cells (Moore et al., 2000). Gene products involved in electron transport were also identified in this dataset. To date, three genes have been identified as belonging to the cytochrome oxidase subunit protein superfamily and we have identified two of these subunits which participate in electron transport and possess transmembrane domains. Another cluster was homologous to the 14-3-3b protein from Meloidugyne incognita (Jaubert et al., 2004) and this regulatory protein has been shown to be important in the development of other parasites.

A L3-specific gene has significant alignment to Glyceraldehyde 3-phosphate dehydrogenase (GAPDH). This enzyme (EC 1.2.1.9) acts as a catalyst during glycolysis. Onchocerca volvulus is a filarial parasite where the L3 is the infective stage (transmitted via the bite of black flies into the circulation of the human host). The GAPDH protein in O. volvulus, which is also up-regulated during the L3 stage, shares sequence identity with the antigens that have been reported to protect against $O$. volvulus infections (Erttmann et al., 2005). Methyl Malonyl CoA Mutase, UDP-Glucuronosyl Transferase, NADH hydrogenase subunit and Peptidylglycine-hydroxylating monooxygenase are some of the other prominent functional categories.

Using GO terms as an alternative functional classification of the 36 genes, 21 genes were identified that could be assigned GO mappings were identified. Of the 19 that had an assignment in biological process, 15 were classified as cellular process (GO:0009987). By molecular function, of the 19 genes that had an assignment, 15 were classified as catalytic activity (GO:0003824) and by cellular component, of the nine genes that had an assignment, four were classified as intracellular part (GO:0044424). Among the genes expressed in the L3, seven do not share homology with other known protein sequences and are novel. Of these, five genes have putative signal peptides or transmembrane domains. As ES products have been implicated in maintaining a positive environment for the parasite, and are intriguing from the vaccine development point of view, these genes warrant further investigation.

A total of 71 genes were identified to be L4-enriched and 58 of these genes have significant alignment to confirmed or hypothetical proteins from other nematodes (Table 5). The gene group enriched with the most number of ESTs (305) among the L4-specific genes is the collagen protein group. The nematode cuticle is made up of collagen proteins that are known to be differently expressed by stage (Elling et al., 2007; Mitreva et al., 2004c). The cuticle of parasitic nematodes has an important role as an interface between the parasite and host and therefore, these genes could potentially provide targets for intervention strategies. The other abundant functional groups are ribosomal proteins, SXC1 protein and the $17 \mathrm{kDa}$ ES antigen protein. Among the genes enriched in the L4 stage, ten do not share sequence identity with other known protein sequences and are novel. Of these, only one gene has signal peptides or transmembrane domain predictions.

Of the 71 L 4 genes, 40 were assigned GO terms. Of 33 that had an assignment in biological process, 19 were involved with cellular metabolic process (GO:0044237). By molecular function, of the 35 that had an assignment, 20 were assigned to structural molecular activity (GO:0005198) and by cellular component, of the 31 that had an assignment, the most abundant was intracellular part (GO:0044424), which constituted 26 of the assignments.

\section{Conclusion}

This study is the first large-scale survey of the larval and adult $O$. ostertagi transcribed genomes. The generation and analysis of the over 2300 genes presented here is a valuable addition to resources for the study of parasitic worms, laying a foundation for further comparative studies on the biology, parasitism and evolution of nematodes. The $O$. ostertagi EST sequencing data have been submitted to public databases and the functional annotations are available online (www.nematode.net) (Martin et al. 2009). This information is therefore accessible as a resource to researchers working on parasitic nematodes for studies, including but not limited to microarrays, RTPCR, RNA interference screens and proteomic experiments. Such studies will aid in the identification of genes involved in host recognition, infection, migration and immune evasion as well as the characterization of targets for vaccines and drugs.

\section{Acknowledgements}

Ostertagia ostertagi EST sequencing at Washington University was supported by NIH-NIAID grant AI 46593 
to MM. The authors would like to thank Claire Murphy and Mike Dante for technical assistance.

\section{Appendix A. Supplementary data}

Supplementary data associated with this article can be found, in the online version, at doi:10.1016/j.vetpar. 2009.02.023.

\section{References}

Apweiler, R., Attwood, T.K., Bairoch, A., Bateman, A., Birney, E., Biswas, M., Bucher, P., Cerutti, L., Corpet, F., Croning, M.D., Durbin, R., Falquet, L., Fleischmann, W., Gouzy, J., Hermjakob, H., Hulo, N., et al., 2001. The InterPro database, an integrated documentation resource for protein families, domains and functional sites. Nucl. Acids Res. 29, 37-40.

Audic, S., Claverie, J.M., 1997. The significance of digital gene expression profiles. Genome Res. 7, 986-995.

Blaxter, M.L., De Ley, P., Garey, J.R., Liu, L.X., Scheldeman, P., Vierstraete, A., Vanfleteren, J.R., Mackey, L.Y., Dorris, M., Frisse, L.M., Vida, J.T., Thomas, W.K., 1998. A molecular evolutionary framework for the phylum Nematoda. Nature 392, 71-75

Burnell, A.M., Houthoofd, K., O'Hanlon, K., Vanfleteren, J.R., 2005. Alternate metabolism during the dauer stage of the nematode Caenorhabditis elegans. Exp. Gerontol. 40, 850-856.

Elling, A., Mitreva, M., Recknor, J., Gai, X., Martin, J., Maier, T., McDermott, J., Hewezi, T., McK Bird, D., Davis, E., Hussey, R., Nettleton, D., McCarter, J., Baum, T., 2007. Divergent evolution of arrested development in the dauer stage of Caenorhabditis elegans and the infective stage of Heterodera glycines. Genome Biol. 8, R211.

Erttmann, K.D., Kleensang, A., Schneider, E., Hammerschmidt, S., Büttner, D.W., Gallin, M., 2005. Cloning, characterization and DNA immunization of an Onchocerca volvulus glyceraldehyde-3-phosphate dehydrogenase (Ov-GAPDH). Biochim. Biophys. Acta (BBA) - Mol. Basis Dis. 1741, 85-94.

Geldhof, P., Claerebout, E., Knox, D., Vercauteren, I., Looszova, A., Vercruysse, J., 2002. Vaccination of calves against Ostertagia ostertagi with cysteine proteinase enriched protein fractions. Parasite Immunol. 24, 263-270.

Gish, W., 1996-2002, www.blast.wustl.edu.

Jaubert, S., Laffaire, J.B., Ledger, T.N., Escoubas, P., Amri, E.Z., Abad, P., Rosso, M.N., 2004. Comparative analysis of two 14-3-3 homologues and their expression pattern in the root-knot nematode Meloidogyne incognita. Int. J. Parasitol. 34, 873-880.

Kall, L., Krogh, A., Sonnhammer, E.L.L., 2004. A combined transmembrane topology and signal peptide prediction method. J. Mol. Biol. 338, 1027-1036.

Kanehisa, M., Goto, S., 2006. KEGG: kyoto encyclopedia of genes and genomes. Nucl. Acids Res. 28, 27-30.

Kotze, A.C., Dobson, R.J., Chandler, D., 2006. Synergism of rotenone by piperonyl butoxide in Haemonchus contortus and Trichostrongylus colubriformis in vitro: potential for drug-synergism through inhibition of nematode oxidative detoxification pathways. Vet. Parasitol. 136, 275-282.

Martin, J., Abubucker, S., Wylie, T., Yin, Y., Wang, Z., Mitreva, M., 2009. Nematode.net update 2008: improvements enabling more efficient data mining and comparative nematode genomics. Nucl. Acids Res. 37, D571-578.

McCarter, J., Dautova Mitreva, M., Martin, J., Dante, M., Wylie, T., Rao, U., Pape, D., Bowers, Y., Theising, B., Murphy, C.V., Kloek, A.P., Chiapelli, B.J., Clifton, S.W., Bird, M.D., Waterston, R., 2003. Analysis and functional classification of transcripts from the nematode Meloidogyne incognita. Genome Biol. 4 R26: 21-19.

Medica, D.L., Sukhdeo, M.V., 1997. Role of lipids in the transmission of the infective stage (L3) of Strongylus vulgaris (Nematoda: Strongylida). J. Parasitol. 83, 775-779.

Mitreva, M., Elling, A.A., Dante, M., Kloek, A.P., Kalyanaraman, A., Aluru, S., Clifton, S.W., Bird, D.M., Baum, T.J., McCarter, J.P., 2004a. A survey of SL1-spliced transcripts from the root-lesion nematode Pratylenchus penetrans. Mol. Gen. Genom. 272, 138-148.

Mitreva, M., Jasmer, D.P., Appleton, J., Martin, J., Dante, M., Wylie, T., Clifton, S.W., Waterston, R.H., McCarter, J.P., 2004b. Gene discovery in the adenophorean nematode Trichinella spiralis: an analysis of transcription from three life cycle stages. Mol. Biochem. Parasitol. 137, 277-291.

Mitreva, M., McCarter, J.P., Arasu, P., Hawdon, J., Martin, J., Dante, M., Wylie, T., Xu, J., Stajich, J.E., Kapulkin, W., Clifton, S.W., Waterston, R.H., Wilson, R.K., 2005. Investigating hookworm genomes by comparative analysis of two Ancylostoma species. BMC Genom. 6, 58.

Mitreva, M., McCarter, J.P., Martin, J., Dante, M., Wylie, T., Chiapelli, B., Pape, D., Clifton, S.W., Nutman, T.B., Waterston, R.H., 2004c. Comparative genomics of gene expression in the parasitic and free-living nematodes Strongyloides stercoralis and Caenorhabditis elegans. Genome Res. 14, 209-220.

Moore, J., Tetley, L., Devaney, E., 2000. Identification of abundant mRNAs from the third stage larvae of the parasitic nematode, Ostertagia ostertagi. Biochem. J. 347, 763-770.

Newton, S.E., Meeusen, E.N.T., 2003. Progress and new technologies for developing vaccines against gastrointestinal nematode parasites of sheep. Parasite Immunol. 25, 283-296.

Prufer, K., Muetzel, B., Do, H.-H., Weiss, G., Khaitovich, P., Rahm, E., Paabo, S., Lachmann, M., Enard, W., 2007. FUNC: a package for detecting significant associations between gene sets and ontological annotations. BMC Bioinformatics 8, 41.

Riddle, D.L., Albert, P.S., 1997. Genetic and environmental regulation of dauer larva development. In: Riddle, D.L., Blumenthal, T., Meyer, B.J., Priess, J.R. (Eds.), C. elegans II. Cold Spring Harbor Laboratory Press, Plainview, pp. 739-768.

The Gene Ontology, C., 2008. The Gene Ontology project in 2008. Nucl. Acids Res. 36, D440-444.

Thompson, F.J., Mitreva, M., Barker, G.L., Martin, J., Waterston, R.H., McCarter, J.P., Viney, M.E., 2005. An expressed sequence tag analysis of the life-cycle of the parasitic nematode Strongyloides ratti. Mol. Biochem. Parasitol. 142, 32-46.

Vercauteren, I., Geldhof, P., Vercruysse, J., Peelaers, I., Van Den Broeck, W., Gevaert, K., Claerebout, E., 2004. Vaccination with an Ostertagia ostertagi polyprotein allergen protects calves against homologous challenge infection. Infect. Immun. 72, 2995-3001.

Visser, A., Geldhof, P., De Maere, V., Knox, D.P., Vercruysse, J., Claerebout, E., 2006. Efficacy and specificity of RNA interference in larval lifestages of Ostertagia ostertagi. Parasitology 133, 777-783.

Wasmuth, J.D., Blaxter, M.L., 2004. prot4EST: translating expressed sequence tags from neglected genomes. BMC Bioinformatics 5, 187.

Wolstenholme, A.J., Fairweather, I., Prichard, R., von Samson-Himmelstjerna, G., Sangster, N.C., 2004. Drug resistance in veterinary helminths. Trends Parasitol. 20, 469-476. 